\title{
ANXA9 as a novel prognostic biomarker associated with immune infiltrates in gastric cancer
}

\author{
Tongtong Zhang Equal first author, 1 , Suyang Yu Equal first author, 1 , Shipeng Zhao ${ }^{\text {Corresp. } 1}$ \\ ${ }^{1}$ Department of Gastrointestinal Surgery, The Third Hospital Affiliated to Hebei Medical University, Shijiazhuang, 050000, Hebei, China, Shijiazhuang, \\ China \\ Corresponding Author: Shipeng Zhao \\ Email address: qxsysy@163.com
}

Background. Gastric cancer (GC) is the most prevalent malignancy among the digestive system tumors. Increasing evidence has revealed that lower mRNA expression of ANXA9 is associated with a poor prognosis in colorectal cancer. However, the role of ANXA9 in GC remains largely unknown. Material and Methods. The Gene Expression Profiling Interactive Analysis (GEPIA) and Human Protein Atlas databases were used to investigate the expression of ANXA9 in GC, which was then validated in the four Gene Expression Omnibus (GEO) datasets. The diagnostic value of ANXA9 for GC patients was demonstrated using a receiver operating characteristic (ROC) curve. The correlation between ANXA9 expression and clinicopathological parameters was analyzed in The Cancer Genome Atlas (TCGA) and UALCAN databases. The Kaplan-Meier (K-M) survival curve was used to elucidate the relationship between ANXA9 expression and the survival time of GC patients. We then performed a gene set enrichment analysis (GSEA) to explore the biological functions of ANXA9. The relationship of ANXA9 expression and cancer immune infiltrates was analyzed using the Tumor Immune Estimation Resource (TIMER). In addition, the potential mechanism of ANXA9 in GC was investigated by analyzing its related genes.Results. ANXA9 was significantly up-regulated in GC tissues and showed obvious diagnostic value. The expression of ANXA9 was related to the age, gender, grade, TP53 mutation, and histological subtype of GC patients. We also found that ANXA9 expression was associated with immune-related biological function. ANXA9 expression was also correlated with the infiltration level of $\mathrm{CD}^{+}{ }^{\mathrm{T}}$ cells, neutrophils, and dendritic cells in GC. Additionally, copy number variation (VNV) of ANXA9 occurred in GC patients. Function enrichment analyses revealed that ANXA9 plays a role in the GC progression by interacting with its related genes.Conclusions. Our results provide strong evidence of ANXA9 expression as a prognostic indicator related to immune responses in GC. 
3 Tongtong Zhang ${ }^{1, \#}$, Suyang $\mathrm{Yu}^{1, \#}$, Shipeng $\mathrm{Zhao}^{1, *}$

${ }^{1}$ Department of Gastrointestinal Surgery, The Third Hospital Affiliated to Hebei Medical University,

5 Shijiazhuang, 050000, Hebei, China.

6 \#These authors contributed equally to this work as co-first author.

$7 \quad{ }^{*}$ Corresponding author.

8 Tongtong Zhang: 18631180295@qq.com

9 Suyang Yu: ysyqtt@163.com

10 Shipeng Zhao: qxsysy@163.com

11 Full postal address:

12 No.139, Ziqiang Road, Qiaoxi District, Shijiazhuang City, 050000, Hebei, China. Tel: +86-18631180295 


\section{Abstract}

Background. Gastric cancer (GC) is the most prevalent malignancy among the digestive system tumors. Increasing evidence has revealed that lower mRNA expression of ANXA9 is associated with a poor prognosis in colorectal cancer. However, the role of ANXA9 in GC remains largely unknown.

Material and Methods. The Gene Expression Profiling Interactive Analysis (GEPIA) and Human Protein Atlas databases were used to investigate the expression of ANXA9 in GC, which was then validated in the four Gene Expression Omnibus (GEO) datasets. The diagnostic value of ANXA9 for GC patients was demonstrated using a receiver operating characteristic (ROC) curve. The correlation between ANXA9 expression and clinicopathological parameters was analyzed in The Cancer Genome Atlas (TCGA) and UALCAN databases.

The Kaplan-Meier (K-M) survival curve was used to elucidate the relationship between ANXA9 expression and the survival time of GC patients. We then performed a gene set enrichment analysis (GSEA) to explore the biological functions of ANXA9. The relationship of ANXA9 expression and cancer immune infiltrates was analyzed using the Tumor Immune Estimation Resource (TIMER). In addition, the potential mechanism of ANXA9 in GC was investigated by analyzing its related genes.

Results. ANXA9 was significantly up-regulated in GC tissues and showed obvious diagnostic value. The expression of ANXA9 was related to the age, gender, grade, TP53 mutation, and histological subtype of GC patients. We also found that ANXA9 expression was associated with immune-related biological function. ANXA9 expression was also correlated with the infiltration level of $\mathrm{CD}^{+} \mathrm{T}$ cells, neutrophils, and dendritic cells in GC. Additionally, copy number variation (VNV) of ANXA9 occurred in GC patients. Function enrichment analyses revealed that ANXA9 plays a role in the GC progression by interacting with its related genes. 
43 Conclusions. Our results provide strong evidence of ANXA9 expression as a prognostic indicator related to

44 immune responses in GC.

45 Key words: gastric cancer, ANXA9, prognosis, immune infiltrates, GEO, TCGA

46

47

48

49

50

51

52

53

54

55

56

57

58

59

60

61

62

63

Peer] reviewing PDF | (2021:06:61985:3:1:NEW 11 Nov 2021) 


\section{INTRODUCTION}

65 Gastric cancer (GC), a cause of some of the the deadliest tumors, is the fifth most common cancer worldwide and is most prevalent in East Asian countries, such as Korea, Mongolia, Japan and China, followed by Eastern Europe [1, 2]. In 2015, there were an estimated 679,000 new GC cases and 498,000 deaths from GC in China

[3]. Despite many recent developments in the treatments of GC, including surgery, chemotherapy, radiation and immunotherapy, all of which can be used alone or in combination [4], the 5-year survival rates of patients with high-grade GC remain poor, at just 30-35\% [5, 6]. Moreover, despite substantial improvements in targeted therapies including HER2, EGFR1 and VEGF, there are still many limitations to these therapies [7-9].

Most patients with GC only demonstrate the clinical features of the disease in the advanced stage which is too

late to receive effective therapy [10]. Therefore, screening novel biomarkers to increase early diagnosis and

treatment of GC is an urgent necessity.

The Annexin A (ANXA) family is known to be related to the progression of cancer [11-13]. Annexin A9

(ANXA9), initially known as annexin 31, is mainly involved in the organization and regulation of membrane/cytoskeleton linkage [14], and is a protein encoding gene located in human chromosome 1q21 [15-

16]. ANXA9 is associated with relapse in both bone and breast cancers [17]. Previous studies have also found that ANXA9 is a potential prognosis indicator for $\mathrm{ER}^{+}$breast cancer and colorectal cancer [18-20].

Furthermore, ANXA9 might be related to the invasion and metastasis of colorectal cancer through the regulation of ADAM17, MMP-9, TIMP-1 and E-cadherin [20]. ANXA9 also participates in the pathogenesis of HPV-Negative head and neck squamous cell carcinomas [21], and plays a key role in the cisplatin-resistance of ovarian carcinoma cells [22]. Importantly, ANXA9 has been discovered to be associated with the 
associations, the potential role and mechanisms of ANXA9 in GC progression and immunology remain unclear.

In the present study, we aimed to systematically assess the role of ANXA9 in GC. First, the ANXA9 expression in GC was investigated using the data from the Cancer Genome Atlas (TCGA, https://portal.gdc.cancer.gov/) [24] and the Gene Expression Omnibus (GEO, https://www.ncbi.nlm.nih.gov/geo/) databases [25]. Next, we explored the correlations between ANXA9 expression and clinicopathologic features, prognosis and immune cell infiltration. We also analyzed the functions and regulating mechanisms of ANXA9. These analyses will help provide a better understanding of a possible molecular mechanism of GC and may help improve treatment for GC.

\section{MATERIALS \& METHODS}

\section{Date acquisition and description}

All the publicly available transcriptome data and clinical information of GC patients we used in this analysis was downloaded from the TCGA database. The TCGA transcriptome data comprised of 375 tumor tissue samples and 32 normal tissue samples.

Microarray series (GSE13861, GSE13911, GSE19826 and GSE79973) containing GC and normal samples were extracted from the GEO database. The GSE13861 dataset, based on the GPL6884 platform, contained 65 GC samples and 19 normal samples. GSE13911 is a microarray dataset that contained 38 patients with GC and 31 normal individuals. The GSE19826 dataset included 12 GC samples and 15 normal samples. 20 samples were acquired from the GSE79973 dataset including $10 \mathrm{GC}$ and 10 normal samples. The GSE13911, GSE19826, and GSE79973 datasets are all from the GPL570 platform.

\section{Gene expression profiling interactive analysis (GEPIA) database analysis}


106 GEPIA (http://gepia.cancer-pku.cn/) is an online database containing 9736 tumors tissue samples and 8587

107

108

109

110

111

112

113

114

115

116

117

normal tissue samples of the TCGA and Genotype-Tissue Expression (GTEx) databases [26]. The expression of ANXA9 was examined in 33 different types of cancers within the "Single Gene Analysis" module of the GEPIA database. A $P$-value less than 0.05 was considered significant.

\section{Validation of ANXA9 expression in the TCGA database and four GEO datasets}

ANXA9 expression was further demonstrated in the TCGA database and four GEO datasets mentioned above.

The Wilcoxon sign-ranked test (Wilcox.test) was used to analyze the difference in the expression of ANXA9 between GC and normal tissue samples. By conducting the receiver operating characteristic (ROC) analysis with "survivalROC" R package to evaluate the diagnostic value discriminating GC from normal tissues [27], we calculated the area under the curve (AUC) of the ANXA9 expression level.

\section{Human Protein Atlas (HPA)}

The The Human Protein Atlas (HPA) database (https://www.proteinatlas.org/) collected the tissue and cell distribution information of 26000 human proteins [28]. In this study, the protein expression of ANXA9 in both GC and normal tissues were acquired from the HPA database.

\section{UALCAN database analysis}

The UALCAN database (http://ualcan.path.uab.edu) is a comprehensive web resource for analyzing TCGA gene expression data [29]. We analyzed the relationship of ANXA9 expression and the clinicopathological characteristics of GC patients with the UALCAN database. The differences of the different groups were compared using the Wilcox.test.

\section{Survival analysis}

After eliminating the 26 samples with missing clinical information, the remaining 349 GC patients in the 
127 TCGA database were ranked into two groups based on the expression of ANXA9-a ANXA9 high expression

128 group and a ANXA9 low expression group. To analyze the overall survival (OS), 3-year survival rate, and 5-

129 year survival rate of GC patients between the different groups, we performed the Kaplan-Meier (K-M) analysis

130 with the log-rank test. Results where $P<0.05$ were considered statistically significant.

131 Functional enrichment analysis

132 All the genes between the ANXA9 high and low expression groups were utilized to perform a gene set

enrichment analysis (GSEA). The terms with a $P$ value $<0.05$ were considered significantly enriched. The

relative expression of 29 immune-related gene sets acquired from the literatures were calculated with single-

sample gene set enrichment analysis (ssGSEA) in the "gsva" R package [30].

Tumor immunity estimation resource (TIMER) database analysis

The TIMER database is a comprehensive resource that we used for the systematic analysis of immune

infiltration levels in different types of cancer [31]. The TIMER database contained macrophages $\mathrm{CD} 4^{+} \mathrm{T}$ cells,

B cells, neutrophils, CD8 ${ }^{+} \mathrm{T}$ cells, and dendritic cells. The "gene" module of TIMER was used to analyze the

correlations between ANXA9 expression and the 6 infiltrating immune cells in GC.

\section{The analysis of ANXA9-related genes}

The differential expression analysis of the ANXA9 high and low expression groups was implemented using

the "Limma" package [32]. The differentially expressed genes (DEGs) were screened with the selection 
with ANXA9.

The ANXA9-related genes were added to the Database for Annotation, Visualization and Integrated Discovery (DAVID) online tool (https://david.ncifcrf.gov/) for GO and KEGG enrichment analysis.

\section{Statistical analysis}

All data in this study was analyzed using R software. The relationship of ANXA9 among clinicopathological characteristics in GC patients was compared using the Wilcox.test. The survival analysis of different groups was performed using the K-M analysis with the log-rank test. In all analyses, $P$ values $<0.05$ were considered statistically significant.

\section{RESULTS}

\section{The expression and diagnostic value of ANXA9 in GC}

To investigate the differences of ANXA9 expression between tumor and normal samples, the ANXA9 mRNA expression levels in 33 human cancers were examined using the GEPIA database. The results showed that ANXA9 expression was significantly increased in breast invasive carcinoma (BRCA), colon adenocarcinoma (COAD), stomach adenocarcinoma (STAD), and rectum adenocarcinoma (READ) compared with the normal tissues. However, low expression of ANXA9 was found in esophageal carcinoma (ESCA), head and neck squamous cell carcinoma (HNSC), kidney chromophobe $(\mathrm{KICH})$, kidney renal clear cell carcinoma (KIRC), kidney renal papillary cell carcinoma (KIRP), skin cutaneous melanoma (SKCM), testicular germ cell tumors (TGCT), uterine corpus endometrial carcinoma (UCEC), and uterine carcinosarcoma (UCS) (Figure 1A, $\boldsymbol{P}<\mathbf{0 . 0 5}$ ). ANXA9 expression in GC and normal samples was analyzed using the transcriptome data from the TCGA database. Consistently, these results revealed that ANXA9 expression was in GC patients

(Figure 1B, $\boldsymbol{P}<\mathbf{0 . 0 5}$ ). The expression of ANXA9 was also analyzed in four GEO microarray series, and the 
results indicated a large increase of ANXA9 expression in GC patients (Figure 1C-F, $\boldsymbol{P}<\mathbf{0 . 0 5}$ ). Based on the data from the HPA database, deeper staining levels of ANXA9 was observed in GC tissues compared to that of normal tissues, revealing a higher protein expression in GC (Figure 1G-J).

Importantly, ROC curves showed that the AUC of the ANXA9 expression levels reached $0.655,0.828,0.645$, 0.809 and 0.774 in the TCGA, GSE13861, GSE13911, GSE19826, and GSE79973 datasets, respectively, suggesting that ANXA9 expression levels could be used to distinguish GC patients from normal individuals

(Figure 2A-E). Collectively, these results illustrated that ANXA9 was both elevated in GC and has diagnostic value, suggesting that ANXA9 has an important regulatory role in the progression of GC.

\section{Relationship of ANXA9 expression and clinicopathological characteristics of GC patients}

Having demonstrated that the expression of ANXA9 was elevated in GC patients, we then explored the relationship of ANXA9 expression and clinicopathological characteristics in GC patients. The results of the TCGA database analysis revealed that 51-70-year old GC patients had a higher ANXA9 level than 71-90-yearold GC patients (Figure 3A, $\boldsymbol{P}<\mathbf{0 . 0 5}$ ). Moreover, an elevated expression of ANXA9 was observed in male GC patients (Figure 3B, $\boldsymbol{P}<\mathbf{0 . 0 5}$ ). ANXA9 expression was not associated, however, with the pathological stage and TNM stage of GC patients in the TCGA database (Figure 3C-F, $\boldsymbol{P}>\mathbf{0 . 0 5}$ ). We also tested the differences in the expression of ANXA9 between Carcinoma diffuse type GC and Adenocarcinoma intestinal type GC based on the Lauren classification. Patients with Adenocarcinoma intestinal type GC had a higher expression level than patients with Carcinoma diffuse type GC (Figure 3G, $\mathrm{P}=0.022$ ).

The relationship between ANXA9 expression and clinicopathological characteristics was analyzed using the UALCAN online database. In the histological subtype analysis, a significant up-regulation of ANXA9 was observed in both IntAdenoNOS and IntAdenoTubular GC patients (Figure $\mathbf{4 A}, \boldsymbol{P}<\mathbf{0 . 0 5}$ ). We also found that 
the expression level of ANXA9 was significantly up-regulated in patients with GC classified as Grade 2 compared with the normal samples (Figure 4B, $\boldsymbol{P}<\mathbf{0 . 0 5}$ ). In addition, increased ANXA9 expression was observed in TP53-mutant GC patients (Figure 4C, $\boldsymbol{P}<\mathbf{0 . 0 5}$ ). However, no significant correlation was found between ANXA9 expression and the age, race, gender, primary tumor, H.pylori infection status, metastasis status, and pathological stage of GC patients (Figure 4D-J, $\boldsymbol{P}>\mathbf{0 . 0 5}$ ). Taken together, these results indicated that high ANXA9 expression is widely correlated with different clinicopathological characteristics of GC.

\section{The prognostic value of ANXA9 in GC}

To further explore whether the ANXA9 was correlated with the survival of patients with GC, we compared the OS between the ANXA9 high expression and ANXA9 low expression groups using the K-M analysis. Notably, the results showed that the GC patients with a high expression of ANXA9 had a higher survival probability (Figure 5A-C, $\boldsymbol{P}<\mathbf{0 . 0 5}$ ), demonstrating that ANXA9 expression was associated with the prognosis of GC patients. We also observed a correlation between ANXA9 expression and OS in other cancers using the UALCAN database. Interestingly, the higher expression of ANXA9 was correlated with lower OS of UCEC patients (Supplementary Figure 1), which was just the opposite of the results for GC patients.

\section{Gene set enrichment analysis}

To investigate the biological functions of ANXA9 in GC, GSEA was carried out to clarify the GO terms and the KEGG pathways that ANXA9 was involved in. Based on the enrichment score, we listed the top 10 most relevant biological processes and pathways. The GO results showed that AXONEMAL DYNEIN COMPLEX ASSEMBLY, AXONEME ASSEMBLY, CILIARY TRANSITION ZONE, CORNIFICATION, CORNIFIED ENVELOPE, INTRACILIARY TRANSPORT, INTRACILIARY TRANSPORT INVOLVED IN CILIUM ASSEMBLY, INTRACILIARY TRANSPORT PARTICLE, KERATINIZATION were enriched in the 
211 ANXA9 high expression group (Figure 6A). Low expression of ANXA9 was associated with immune-related

212 biological functions, including CHEMOKINE BINDING, CYTOSOLIC RIBOSOME, LEUKOCYTE

213 ADHESION TO VASCULAR ENDOTHELIAL CELL, PHAGOCYTIC CUP, POSITIVE REGULATION

214 OF LEUKOCYTE CELL-CELL ADHESION, POSITIVE T CELL SELECTION, RESPONSE TO

215 CHEMOKINE, and T CELL SELECTION (Figure 6B). Interestingly, KEGG analysis demonstrated that the

216 genes in both the ANXA9 high expression and ANXA9 low expression groups participated in STEROID

217 BIOSYNTHESIS, HEMATOPOIETIC CELL LINEAGE, and RIBOSOME signal pathways. The

218 comprehensive analysis of these results indicated that ANXA9 plays a regulatory role in the progression of GC.

219 Correlation between ANXA9 expression and immune infiltration levels in GC

220 Based on the results of the functional enrichment, we observed that ANXA9 is related to the immune response

221 in GC. Therefore, we further calculated the number of immune cells in each GC sample using ssGSEA. 21

222 immune-response gene sets were significantly different between the ANXA9 high expression and ANXA9 low

223 expression groups, These gene sets include APC_co_inhibition, B_cells, CCR, CD8._T_cells, Check.point,

224 Cytolytic_activity, DCs, HLA, Inflammation.promoting, Neutrophils, NK_cells, pDCs, T_cell_co.inhibition,

225 T_cell_co.stimulation, T_helper_cells, Tfh, Th1_cells, Th2_cells, TIL, Treg and Type_II_IFN_Reponse. The

expression of these 21 immune-response gene sets were significantly increased in the ANXA9 low expression

group (Figure 7A, $\boldsymbol{P}<\mathbf{0 . 0 5}$ ). We then investigated the correlation between ANXA9 expression and immune

infiltration in the TIMER database. As shown in Figure 7B, ANXA9 expression was significantly negatively

correlated with the infiltration level of $\mathrm{CD}^{+} \mathrm{T}$ cells $(\mathrm{Cor}=-0.161, P=1.91 \mathrm{e}-03)$, neutrophils $(\mathrm{Cor}=-0.138, P$

$=7.58 \mathrm{e}-03)$, and dendritic cells (Cor $=-0.153, P=3.05 \mathrm{e}-03)$ in $\mathrm{GC}$. 
232

DNA methylation is the main epigenetic mechanism for regulating gene expression in the tumorigenesis and development [33]. We used MEXPRESS to analyze whether the expression of ANXA9 was related to its methylation. Figure 8 showed that there was a negative correlation between the ANXA9 expression and the methylation of $\mathrm{CpG}$ and promoter regions. 4 abnormal methylation sites with cut-off value of correlation coefficient $<-0.3$ were observed including $\operatorname{cg} 25468058$ (Cor $=-0.503), \operatorname{cg} 04144222$ (Cor $=-0.523)$, $\operatorname{Cg} 07337598(\mathrm{Cor}=-0.349)$, and $\operatorname{cg} 13320146(\mathrm{Cor}=-0.321)$. The ANXA9 expression positively correlated with its $\mathrm{CNV}$ change $(\mathrm{Cor}=0.240, \mathrm{P}<0.0001)$. Our results showed that the CNV change of ANXA9 occurred in GC patients.

\section{Analysis of ANXA9-related genes}

To further investigate the role of ANXA9 in GC, we identified 179 ANXA9-related genes with the criteria $\mid$ Cor $\mid>0.3, P<0.05$ based on the DEGs between ANXA9 high and ANXA9 low expression groups (Figure

9A, Supplementary Table 1). From the PPI network, we retrieved 162 significantly positively related genes and 17 significantly negatively related genes, which were utilized for GO and KEGG enrichment analysis

(Figure 9B and 9C). The enriched biological process (BP) terms were actin filament bundle assembly, skeletal muscle contraction, cell-cell adhesion, Rho protein signal transduction, and cellular response to hyperoxia. The ANXA9-related genes were associated with cellular components (CC) terms, such as endoplasmic reticulum membrane, primary cilium, cell-cell adherens junction, extracellular exosome, and endoplasmic reticulum-Golgi intermediate compartment membrane. The molecular function (MF) terms associated with ANXA9-related genes were microtubule motor activity, Rho guanyl-nucleotide exchange factor activity, actin filament binding, and cadherin binding involved in cell-cell adhesion were significantly enriched. However, there was no significant enrichment of KEGG pathways. The data indicated that ANXA9 - 
253

254

255

256

related genes may also play a role in GC progression.

\section{DISCUSSION}

GC is a common malignancy of the digestive system. Although great advances have been made in the diagnosis and treatment of GC, the prognosis of GC patients is still terrible [5]. An increasing number of studies have noted the superiority of molecular therapy in the treatment of GC [34, 35]. Therefore, it is urgent to screen novel biomarkers to increase early diagnosis and treatment of GC.

To the best of our knowledge, our study was the first to systematically explore the role of ANXA9 in GC. We initially investigated the expression of ANXA9 in pan-cancer samples using the GEPIA site, and found that ANXA9 expression was up-regulated in most cancer samples, including GC samples, when compared to normal samples, as well as GC (Figure 1A). The bioinformatics analysis of data from the TCGA and GEO databases also suggested that ANXA9 expression was significantly increased in GC samples (Figure 1B-G). Interestingly, the analyses we performed using the UALCAN database revealed that ANXA9 expression was significantly related to GC grade, TP53 mutation and histological subtype. More importantly, the K-M analysis suggested that ANXA9 expression was associated with the prognosis of GC patients (Figure 5A-C). Consistent with our results, it has been suggested that ANXA9 is associated with the prognosis of breast cancer and colorectal cancer [18-20]. ANXA9 could also affect the pathogenesis of HPV-Negative head and neck squamous cell carcinomas [21] and regulate the enhanced cisplatin-resistance in ovarian carcinoma [22].

Notably, ANXA9 expression was higher in GC samples compared to normal samples and the high ANXA9 expression group had a better OS rate (Figure 1C-G and Figure 5A-C), which is the opposite of what we see in colorectal cancer [20] and UCEC (Supplementary Figure 1). We speculate that this difference of ANXA9 role among GC, UCEC and colorectal cancer may be due to the dual roles of genes in cancer pathogenesis. For 
274

275

276

277

278

279

280

281

282

283

284

example, reducing NLK expression can suppress cell growth and tumorigenesis in some tumors, but can produce the opposite effects in other tumors [36]. Similarly, YY1 is up-regulated in many cancers, while sometimes is down-regulated in other cancers [37]. PA2G4 also has opposite roles in prostate cancer and colon cancer [38]. On the other hand, this difference of ANXA9 role between GC and colorectal cancer may be caused by the heterogeneity of GC and colorectal cancer. Research suggests that the factors and molecular pathways involved in GC tumorigenesis are different from colorectal cancer [39]. For example, alterations of p53 or missing genes may play different roles in the progression of GC compared to colorectal cancers [40]. In addition, hepatic metastases from GC present more systematic and aggressive tumor behavior than those from colorectal cancer [41]. Furthermore, it has been revealed that MDR1 mRNA levels in GC cells are significantly lower than those in colon cancer cells, which means they have different epigenetic regulations such as DNA methylation and/or histone deacetylation in GC and colon cancer [42]. Particularly, ANXA may play a multifaceted and possibly distinctive role in different cancers. For instance, annexin A7 acts as a tumor suppressor in prostate cancer, melanoma and glioblastoma [43-45], whereas annexin A7 can accelerate the progression of liver, colorectal, and breast cancers [46-48]. Thus, given the expression pattern and prognostic impact of ANXA9, we speculated that the ANXA9 might not be related to the occurrence of GC, but might affect the development of GC. Namely, the occurrence of GC might induce the up-regulation of ANXA9 expression resulting in a protective effect on human body. It also has been noted that CA12 expression is higher in breast cancer tissues and a high CA12 expression in breast cancer patients is associated with better OS [49]. In addition, Li et al. also found that BUB1 was highly expressed in gastric cancer compared with normal gastric tissue and a K-M survival analysis showed that the OS time was prolonged in gastric cancer patients with a high expression of BUB1 [50]. Collectively, these findings suggested that ANXA9 might play 
295

296

297

298

299

300

301

302

303

304

305

306

307

308

309

310

311

312

313

314

315

an important role in GC, and that ANXA9 could be selected as a biomarker for the diagnosis and treatment of GC.

The genes in ANXA9 high expression group were mainly enriched in an axonemal dynein complex (Figure 6A). It has been suggested that ANXA9 is found in protein complexes containing periplakin, a cytoskeletal linker protein belonging to the plakin family [15]. Moreover, ANXA9 has been reported to participate in the bone metastasis of breast cancer. Thus, we speculated that ANXA9 might be involved in the bone metastasis of GC. On the other hand, the genes in ANXA9 low expression group were primarily involved in immune-related biological processes, such as chemokine binding and $\mathrm{T}$ cell selection (Figure 6B). Therefore, we speculated that ANXA9 might also regulate GC prognosis by influencing the immune response. Consistent with our hypothesis, patients in ANXA9 low expression group showed higher proportions of infiltrating immune cells (Figure 7A), indicating that the poor OS of patients in the ANXA9 low expression group might be caused by immunosuppressive milieu [51] and increasing the expression of ANXA9 could protect against the immunosuppressive environment of GC resulting in an improved GC prognosis [50]. Notably, we found that ANXA9 expression was significantly negatively correlated with the infiltration level of CD8+ T cells (Figure 7B). It has been suggested that CD8+ $\mathrm{T}$ cells could produce interferon Gamma (IFNG) and introduce the expression of PD-1/PD-L1, resulting in antitumor immunity [52]. Therefore, these finding implies that the ANXA9 low expression group might have a stronger tumor immunosuppressive environment.

We further investigated the DNA methylation level of ANXA9 and the correlation between CNV and expression of ANXA9 expression, and found that there was a negative correlation between ANXA9 expression and the methylation of $\mathrm{CpG}$ and promoter regions, and ANXA9 expression positively correlated with its CNV (Figure 8). Therefore, the DNA methylation and CNV might affect the expression of ANXA9. 
316 Ultimately, we also found ANXA9 might affect the survival of GC by affecting ANXA9-related genes, which

317 can regulating actin filament bundle assembly, skeletal muscle contraction, cell-cell adhesion, Rho protein

318 signal transduction, and cellular response to hyperoxia.

\section{CONCLUSION}

320

321

322

323

324

325

326

327

328

329

330

331

332

333

334

This study found that ANXA9 expression significantly increased in GC samples compared to normal tissues and the ANXA9 high expression group showed a higher survival probability. Moreover, the present study also revealed that increasing the expression of ANXA9 might improve GC prognosis by protecting against the immunosuppressive environment of GC. Furthermore, the expression change of ANXA9 might be caused by the the DNA methylation and CNV ANAXA9. In a word, this study revealed the potential role of ANXA9 in GC immunology and prognosis, which might contribute to the further understanding of the possible molecular mechanism of GC and lead to improved GC treatments. However, more studies are needed to further illustrate the specific regulatory mechanisms of ANXA9.

\section{Abbreviations}

GC: gastric cancer; GEPIA: Gene Expression Profiling Interactive Analysis; GEO: Gene Expression Omnibus; Wilcox.test: Wilcoxon sign-ranked test; ROC: receiver operating characteristic; TCGA: The Cancer Genome Atlas; TIMER: Tumor Immune Estimation Resource; ANXA: Annexin A; ANXA9: Annexin A9; GTEx: Genotype-Tissue Expression; AUC: area under the curve; HPA: 2.4. Human Protein Atlas; OS: overall survival; K-M: Kaplan-Meier; GSEA: Gene set enrichment analysis; ssGSEA: single-sample gene set enrichment; DEGs: differentially expressed genes; BRCA: Breast Invasive Carcinoma; COAD: Colon Adenocarcinoma; STAD: Stomach Adenocarcinoma; READ: Rectum adenocarcinoma; ESCA: Esophageal carcinoma; HNSC: Head and Neck squamous cell carcinoma; KICH: Kidney Chromophobe; KIRC: Kidney 
renal clear cell carcinoma; KIRP: Kidney renal papillary cell carcinoma; SKCM: Skin Cutaneous Melanoma;

338 TGCT: Testicular Germ Cell Tumors; UCEC: Uterine Corpus Endometrial Carcinoma; UCS: Uterine

339 Carcinosarcoma; CNV: copy number variation

340 Acknowledgments

341 Not applicable.

342 Author Contributions

343 Shipeng Zhao conceived and designed this study. Tongtong Zhang and Suyang Yu collected and analyzed the

344 data, and wrote the paper. Shipeng Zhao revised the manuscript. All authors read and approved the manuscript

345 for publication.

346 Funding

347 This work was not supported by any funding.

348 Data Availability

349 GSE13911, GSE19826, GSE79973 datasets used in the present study can be found in GEO database

350 (https://www.ncbi.nlm.nih.gov/geo/), and the data of TCGA (https://portal.gdc.cancer.gov/) is available.

351 Ethics approval and consent to participate

352 No ethics approval was required for this work. All utilized public data sets were generated by others who 353 obtained ethical approval.

354 Consent for publication

355 Not applicable.

356 Competing Interests

357 The authors have declared that no competing interest exists.

Peer] reviewing PDF | (2021:06:61985:3:1:NEW 11 Nov 2021) 


\section{References}

1. Correa P: Gastric cancer: overview. Gastroenterology Clinics of North America 2016, 42(2):211-217.

2. Tsukanov VV, Butorin NN, Maady AS, Shtygasheva OV, Amelchugova OS, Tonkikh JL, Fassan M, Rugge M: Helicobacter pylori Infection, Intestinal Metaplasia, and Gastric Cancer Risk in Eastern Siberia. Helicobacter 2011, 16(2):107-112.

3. Chen W, Zheng R, Baade PD, Zhang S, Zeng H, Bray F, Jemal A, Yu XQ, He J: Cancer statistics in China, 2015. CA-A Cancer Jurnal for Clinicians 2016, 66(2):115-132.

4. Song $\mathrm{Z}, \mathrm{Wu} \mathrm{Y}$, Yang J, Yang D, Fang X: Progress in the treatment of advanced gastric cancer. Tumour Biology 2017, 39(7):1010428317714626.

5. Miller KD, Nogueira L, Mariotto AB, Rowland JH, Yabroff KR, Alfano CM, Jemal A, Kramer JL, Siegel RL: Cancer treatment and survivorship statistics, 2019. CA-A Cancer Jurnal for Clinicians 2019, 69(5):363-385.

6. Chon SH, Berlth F, Plum PS, Herbold T, Alakus H, Kleinert R, Moenig SP, Bruns CJ, Hoelscher AH, Meyer HJ: Gastric cancer treatment in the world: Germany. Transl Gastroenterol Hepatol 2017, 26(2):53.

7. Kim $\mathrm{C}$, Mulder $\mathrm{K}$, Spratlin $\mathrm{J}$ : How prognostic and predictive biomarkers are transforming our understanding and management of advanced gastric cancer. Oncologist 2014, 19(10):1046-1055.

8. Lordick F, Kang YK, Chung HC, Salman P, Oh SC, Bodoky G, Kurteva G, Volovat C, Moiseyenko VM, Gorbunova V, Park JO, Sawaki A, Celik I, Götte H, Melezínková H, Moehler M; Arbeitsgemeinschaft Internistische Onkologie and EXPAND Investigators: Capecitabine and cisplatin with or without cetuximab for patients with previously untreated advanced gastric cancer (EXPAND): a randomised, open-label phase 3 trial. Lancet Oncology 2013, 14(6):490-499.

9. Maeda K, Chung YS, Ogawa Y, Takatsuka S, Kang SM, Ogawa M, Sawada T, Sowa M: Prognostic value of vascular endothelial growth factor expression in gastric carcinoma. Cancer 1996, 77(5):858-863.

10. Luebeck EG, Curtius K, Jeon J, Hazelton WD: Impact of tumor progression on cancer incidence curves. Cancer Research 2013, 73(3):1086-1096.

11. Boudhraa Z, Bouchon B, Viallard C, D'Incan M, Degoul F: Annexin A1 localization and its relevance to cancer. Clinical Science (Lond) 2016, 130(4):205-220.

12. Qi H, Liu S, Guo C, Wang J, Greenaway FT, Sun MZ: Role of annexin A6 in cancer. Oncology Letters 2015, 10(4):1947-1952.

13. Lauritzen SP, Boye TL, Nylandsted J: Annexins are instrumental for efficient plasma membrane repair in cancer cells. Seminars in Cell \& Developmental Biology 2015, 45:32-38.

14. Morgan RO, Bell DW, Testa JR, Fernandez MP: Human annexin 31 genetic mapping and origin. Gene 1999, 227(1):33-38.

15. Boczonadi V, Määttä A: Annexin A9 is a periplakin interacting partner in membrane-targeted cytoskeletal linker protein complexes. FEBS Letters 2012, 586(19):3090-3096.

16. Goebeler V, Ruhe D, Gerke V, Rescher U: Atypical properties displayed by annexin A9, a novel member of the annexin family of $\mathbf{C a ( 2 + )}$ and lipid binding proteins. FEBS Letters 2003, 546(23):359-364. 
398 17. Smid M, Wang Y, Klijn JG, Sieuwerts AM, Zhang Y, Atkins D, Martens JW, Foekens JA: Genes associated with breast cancer metastatic to bone. Journal of Clinical Oncology 2006, 24(15):22612267.

18. Xiao B, Hang J, Lei T, He Y, Kuang Z, Wang L, Chen L, He J, Zhang W, Liao Y, Sun Z, Li L: Identification of key genes relevant to the prognosis of ER-positive and ER-negative breast cancer based on a prognostic prediction system. Molecular Biology Reports 2019, 46(2):2111-2119.

19. Miyoshi N, Yamamoto H, Mimori K, Yamashita S, Miyazaki S, Nakagawa S, Ishii H, Noura S, Ohue M, Yano M, Doki Y, Mori M: ANXA9 gene expression in colorectal cancer: A novel marker for prognosis. Oncology Letters 2014, 8(5):2313-2317.

20. Yu S, Bian H, Gao X, Gui L: Annexin A9 promotes invasion and metastasis of colorectal cancer and predicts poor prognosis. International Journal of Molecular Medicine 2018, 41(4):2185-2192.

21. Salom C, Álvarez-Teijeiro S, Fernández MP, Morgan RO, Allonca E, Vallina A, Lorz C, de Villalaín L, Fernández-García MS, Rodrigo JP, García-Pedrero JM: Frequent Alteration of Annexin A9 and A10 in HPV-Negative Head and Neck Squamous Cell Carcinomas: Correlation with the Histopathological Differentiation Grade. Journal of Clinical Medicine 2019, 8(2):229.

22. Kou X, Ding H, Li L, Chao H: Hsa-miR-105-1 Regulates Cisplatin-Resistance in Ovarian Carcinoma Cells by Targeting ANXA9. Analytical Cellular Pathology (Amst) 2021, 2021:6662486.

23. Nakashima Y, Nahar S, Miyagi-Shiohira C, Kinjo T, Kobayashi N, Kitamura S, Saitoh I, Watanabe M, Fujita J, Noguchi H: Identification of Proteins Differentially Expressed by Adipose-derived Mesenchymal Stem Cells Isolated from Immunodeficient Mice. International Journal of Molecular Sciences 2019, 20(11):2672.

24. Tomczak K, Czerwińska P, Wiznerowicz M: The Cancer Genome Atlas (TCGA): an immeasurable source of knowledge. Contemp Oncol (Pozn) 2015, 19(1A):A68-77.

25. Edgar R, Domrachev M, Lash AE: Gene expression omnibus: NCBI gene expression and hybridization array data repository. Nucleic Acids Research 2002, 30:207-210.

26. Tang Z, Li C, Kang B, Gao G, Li C, Zhang Z: GEPIA: a web server for cancer and normal gene expression profiling and interactive analyses. Nucleic Acids Research 2017, 45(W1):W98-W102.

27. Heagerty PJ, Lumley T, Pepe MS: Time-dependent ROC curves for censored survival data and a diagnostic marker. Biometrics 2000, 56(2):337-344.

28. Asplund A, Edqvist PH, Schwenk JM, Pontén F: Antibodies for profiling the human proteome-The Human Protein Atlas as a resource for cancer research. Proteomics 2012, 12(13):2067-2077.

29. Chandrashekar DS, Bashel B, Balasubramanya SAH, Creighton CJ, Ponce-Rodriguez I, Chakravarthi BVSK, Varambally S: UALCAN: A Portal for Facilitating Tumor Subgroup Gene Expression and Survival Analyses. Neoplasia 2017, 19(8):649-658.

30. Hänzelmann S, Castelo R, Guinney J: GSVA: gene set variation analysis for microarray and RNA-seq data. BMC Bioinformatics 2013, $14: 7$.

31. Li T, Fan J, Wang B, Traugh N, Chen Q, Liu JS, Li B, Liu XS: TIMER: A Web Server for Comprehensive Analysis of Tumor-Infiltrating Immune Cells. Cancer Research 2017, 77(21):e108-e110.

32. Ritchie ME, Phipson B, Wu D, Hu Y, Law CW, Shi W, Smyth GK: limma powers differential expression analyses for RNA-sequencing and microarray studies. Nucleic Acids Research 2015, 
439

440

441

442

443

444

445

446

447

448

449

450

451

452

453

454

455

456

457

458

459

460

461

462

463

464

465

466

467

468

469

470

471

472

473

474

475

476

477

478

479

43(7):e47.

33. Maeder ML, Angstman JF, Richardson ME, Linder SJ, Cascio VM, Tsai SQ, Ho QH, Sander JD, Reyon D, Bernstein BE, Costello JF, Wilkinson MF, Joung JK: Targeted DNA demethylation and activation of endogenous genes using programmable TALE-TET1 fusion proteins. Nature Biotechnology 2013, 31(12):1137-1142.

34. Xu B, Huang C, Yang X, Li X, Li L, Ding Y: Significance and prognostic role of human epidermal growth factor receptor 2 and RAB1A expression in gastric cancer. Oncology Letters 2018, 15(4):5185-5192.

35. Shitara K, Iwata H, Takahashi S, Tamura K, Park H, Modi S, Tsurutani J, Kadowaki S, Yamaguchi K, Iwasa S, Saito K, Fujisaki Y, Sugihara M, Shahidi J, Doi T: Trastuzumab deruxtecan (DS-8201a) in patients with advanced HER2-positive gastric cancer: a dose-expansion, phase 1 study. Lancet Oncology 2019, 20(6):827-836.

36. Li SZ, Zeng F, Li J, Shu QP, Zhang HH, Xu J, Ren JW, Zhang XD, Song XM, Du RL: Nemo-like kinase (NLK) primes colorectal cancer progression by releasing the E2F1 complex from HDAC1. Cancer Lett 2018, 431:43-53.

37. Hays E, Bonavida B: YY1 regulates cancer cell immune resistance by modulating PD-L1 expression. Drug Resist Updat 2019, 43: 10-28.

38. Nguyen DQ, Hoang DH, Nguyen Vo TT, Huynh V, Ghoda L, Marcucci G, Nguyen LXT: The role of ErbB3 binding protein 1 in cancer: friend or foe? J Cell Physiol 2018, 233:9110-9120.

39. Park SM, Lee J, Kim YA, Chang YJ, Kim MS, Shim YM, Zo JI, Yun YH: Factors related with colorectal and stomach cancer screening practice among disease-free lung cancer survivors in Korea. BMC Cancer 2017, 17(1):600.

40. Kataoka M, Okabayashi T, Johira H, Nakatani S, Nakashima A, Takeda A, Nishizaki M, Orita K, Tanaka N: Aberration of p53 and DCC in gastric and colorectal cancer. Oncol Rep 2000, 7(1):99103.

41. Oguro S, Imamura H, Yoshimoto J, Ishizaki Y, Kawasaki S: Liver metastases from gastric cancer represent systemic disease in comparison with those from colorectal cancer. Journal of HepatoBiliary-Pancreatic Sciences 2016, 23(6):324-32.

42. Lee TB, Park JH, Min YD, Kim KJ, Choi CH: Epigenetic mechanisms involved in differential MDR1 mRNA expression between gastric and colon cancer cell lines and rationales for clinical chemotherapy. BMC Gastroenterol 2008, 8:33.

43. Srivastava M, Bubendorf L, Srikantan V, Fossom L, Nolan L, Glasman M, Leighton X, Fehrle W, Pittaluga S, Raffeld M, Koivisto P, Willi N, Gasser TC, Kononen J, Sauter G, Kallioniemi OP, Srivastava S, Pollard HB: ANX7, a candidate tumor suppressor gene for prostate cancer. Proceedings of the national academy of sciences of the United States of America 2001, 98(8):45754580.

44. Hung KS, Howng SL: Prognostic significance of annexin VII expression in glioblastomas multiforme in humans. Journal of Neurosurgery 2003, 99(5):886-892.

45. Kataoka TR, Ito A, Asada H, Watabe K, Nishiyama K, Nakamoto K, Itami S, Yoshikawa K, Ito M, Nojima H, Kitamura Y: Annexin VII as a novel marker for invasive phenotype of malignant melanoma. Japanese Journal of Cancer Research 2000, 91(1):75-83. 
480 46. Srivastava M, Bubendorf L, Raffeld M, Bucher C, Torhorst J, Sauter G, Olsen C, Kallioniemi OP, 481 Eidelman O, Pollard HB: Prognostic impact of ANX7-GTPase in metastatic and HER2-negative 482 breast cancer patients Clinical Cancer Research 2004, 10(7):2344-2350.

47. Srivastava M, Torosyan Y, Raffeld M, Eidelman O, Pollard HB, Bubendorf L: ANXA7 expression represents hormone-relevant tumor suppression in different cancers International Journal of Cancer 2007, 121(12):2628-36.

48. Sun MZ, Liu S, Tang J, Wang Z, Gong X, Sun C, Greenaway F: Proteomics analysis of two mice hepatocarcinoma ascites syngeneic cell lines with high and low lymph node metastasis rates provide potential protein markers for tumor malignancy attributes to lymphatic metastasis. Proteomics 2009, 9(12):3285-3302.

49. Li Y, Lei B, Zou J, Wang W, Chen A, Zhang J, Fu Y, Li Z: High expression of carbonic anhydrase 12 (CA12) is associated with good prognosis in breast cancer. Neoplasma 2019, 66(3):420-426.

52. Ribas A, Hu-Lieskovan S: What does PD-L1 positive or negative mean? Journal of Experimental Medicine 2016, 213(13):2835-2840.

50. $\mathrm{Li} \mathrm{X}, \mathrm{He} \mathrm{J}, \mathrm{Yu} \mathrm{M}$, Zhang $\mathrm{W}$, Sun $\mathrm{J}$ : [BUB1 gene is highly expressed in gastric cancer:analysis based on Oncomine database and bioinformatics]. Nan Fang Yi Ke Da Xue Xue Bao 2020, 40(5):683-692.

51. Cao R, Yuan L, Ma B, Wang G, Tian Y: Immune-related long non-coding RNA signature identified prognosis and immunotherapeutic efficiency in bladder cancer (BLCA). Cancer Cell International 2020, 20:276.

499 
Figure 1

Figure 1

The expression level of ANXA9 in pan-cancer and GC. A: the ANXA9 mRNA levels in 33 human cancers. B: the ANXA9 mRNA level in GC was up-regulated compared to the normal tissues based on the TCGA database. C-F: the ANXA9 mRNA level in GC was up-regulated compared to normal tissues based on GSE13861, GSE13911, GSE19826, and GSE79973. G: the ANXA9 protein expression in GC was higher than in the normal tissues based on the HPA database. G-H: the ANXA9 protein expression in GC from the HPA database. I-J: the ANXA9 protein expression in normal samples from the HPA database.
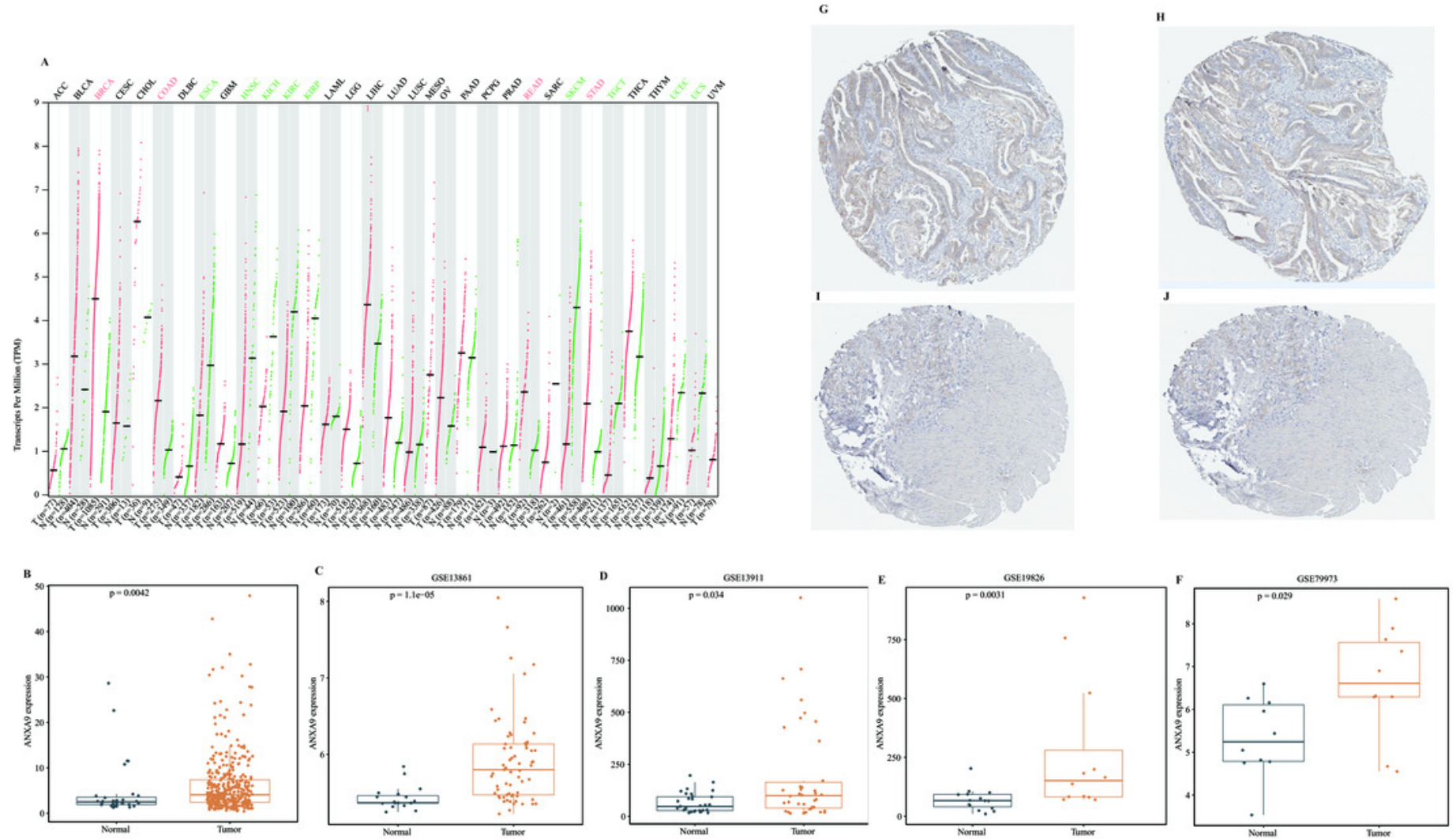
Figure 2

Figure 2

ROC curves showed that ANXA9 could distinguish the GC patients from normal individuals. A-

E: ROC curves in the TCGA database (A) and GSE13861 (B), GSE13911 (C), GSE19826 (D), and GSE79973 (E) datasets. 

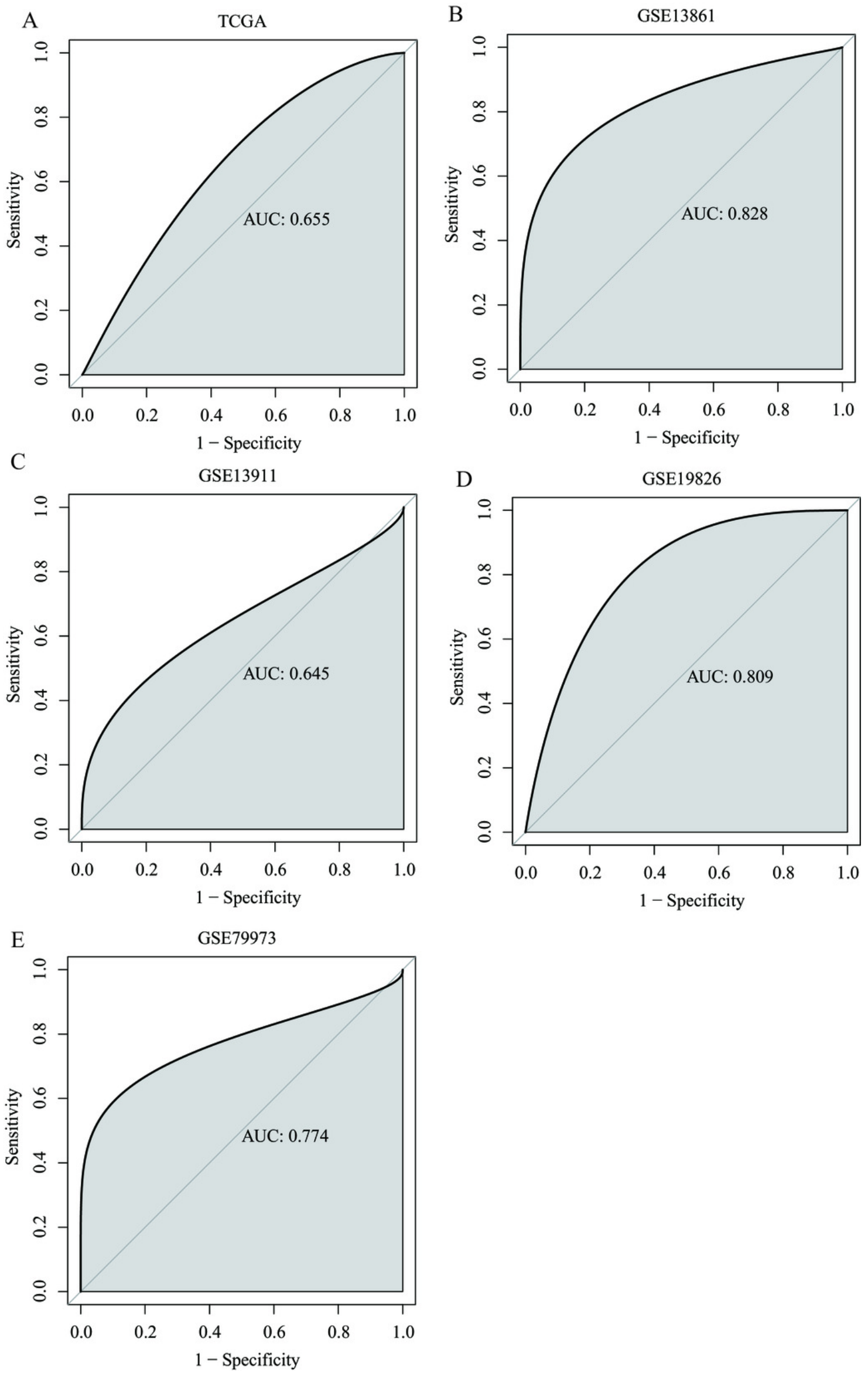


\section{Figure 3}

Figure 3

Relationship of ANXA9 expression and clinicopathological characteristics of GC patients based on the TCGA database. A-F: the mRNA expression levels by age (A), gender (B), tumor stage (C), pathological T stage (D), pathological $N$ stage $(E)$, pathological $M$ stage $(F)$, and Lauren classification $(G)$ based on the TCGA database. 


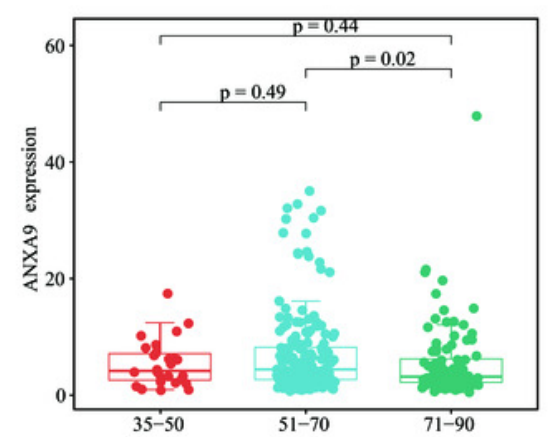

D

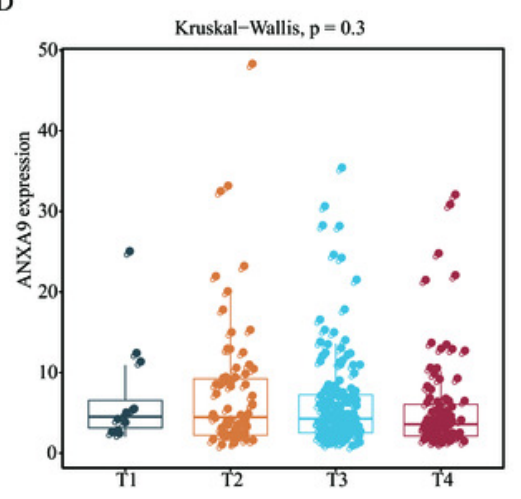

B

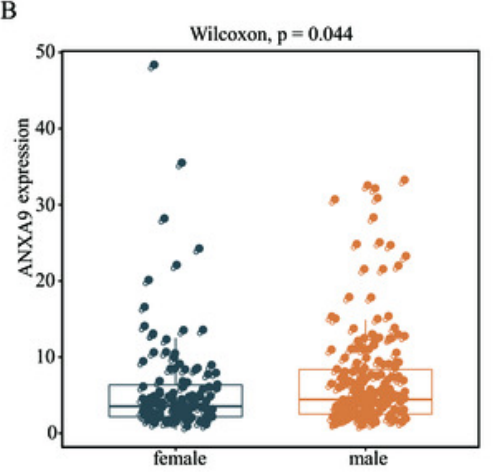

E

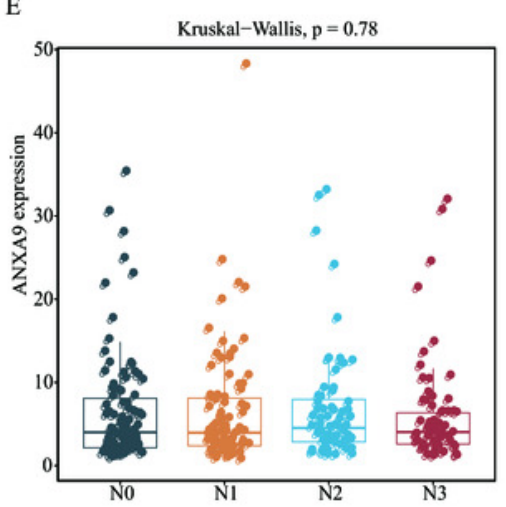

G

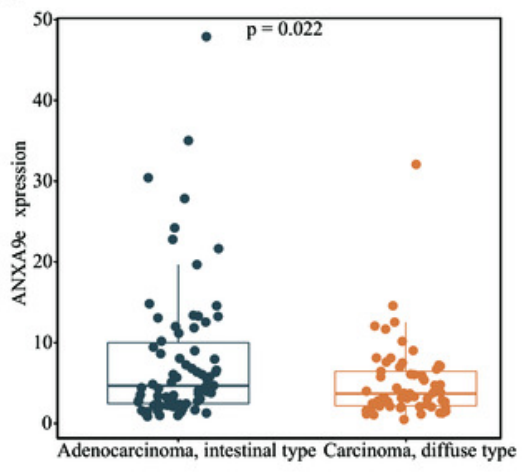

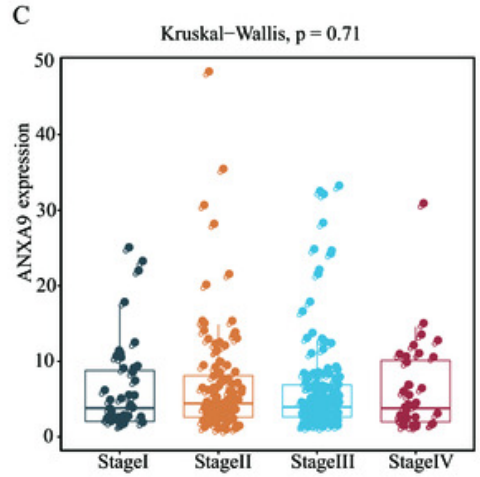

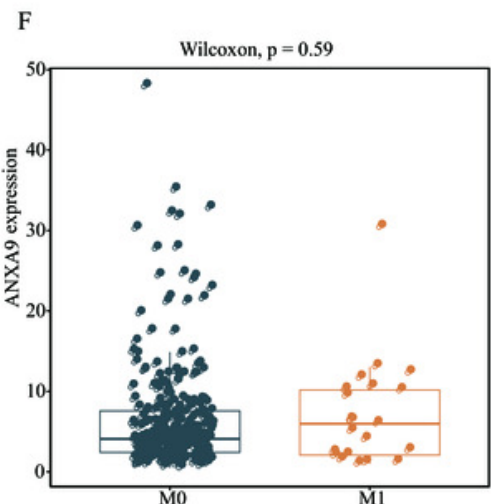




\section{Figure 4}

Figure 4

Relationship of ANXA9 expression and clinicopathological characteristics of GC patients based on the UALCAN online database. A-I: the mRNA expression levels between different histological subtypes (A), tumor grade (B), TP53 mutation status (C), age (D), race (E), gender (F), sample types (G), H.pylori infection status (H), nodal metastasis status (I), and tumor stage based on the UALCAN online database. 

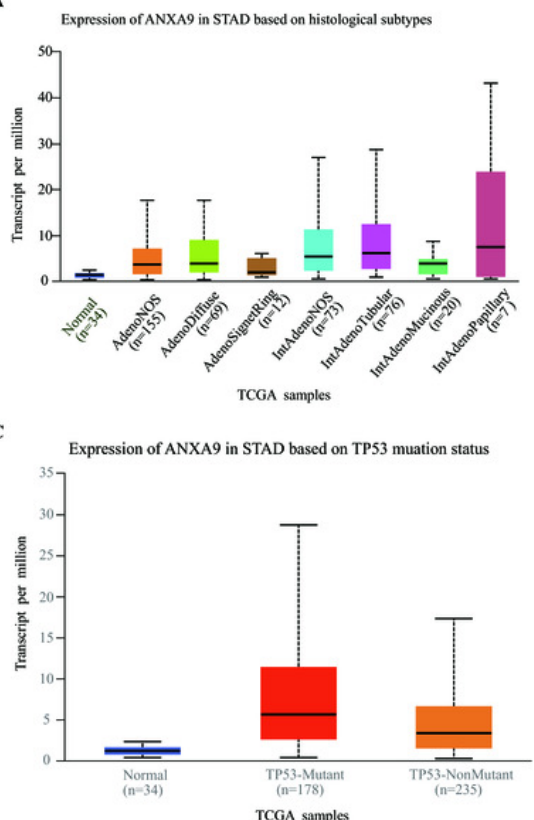

$\mathbf{E}$
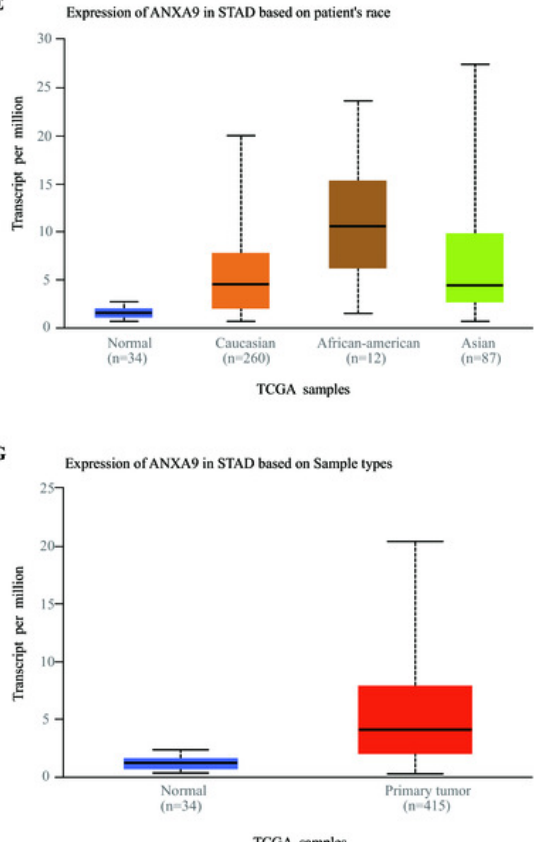

I

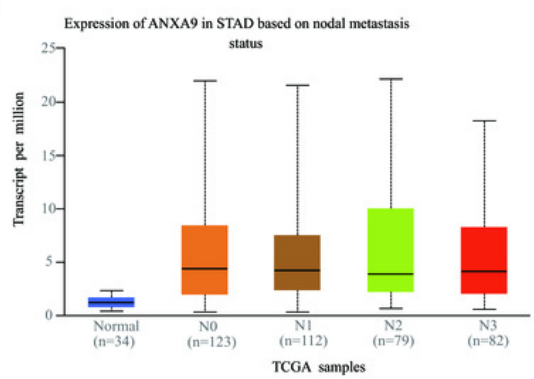

B

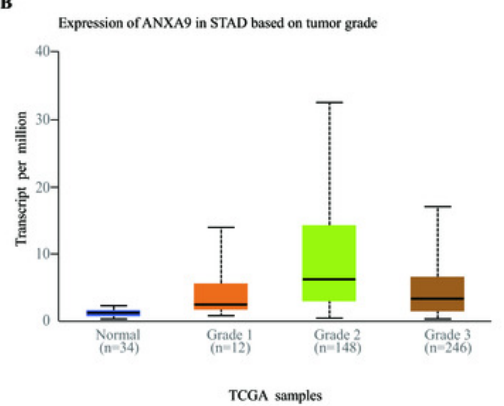

D
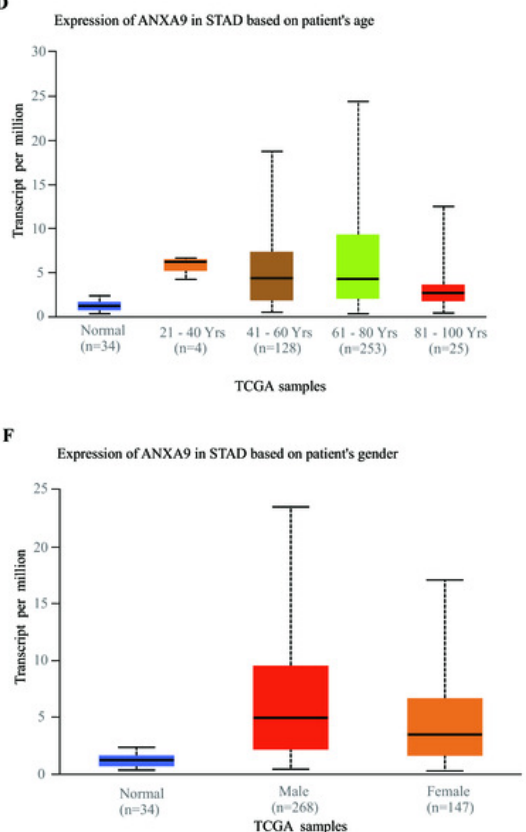

H Expression of ANXA9 in STAD based on H.pylori infection

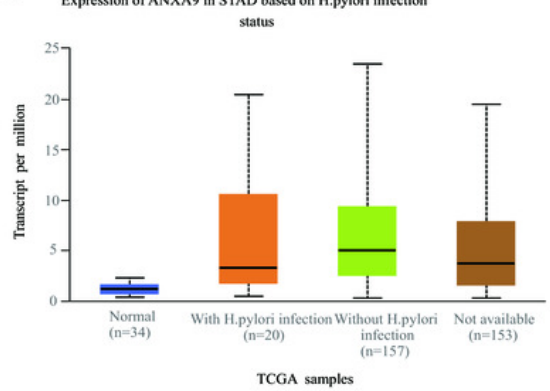

$\mathbf{J}$

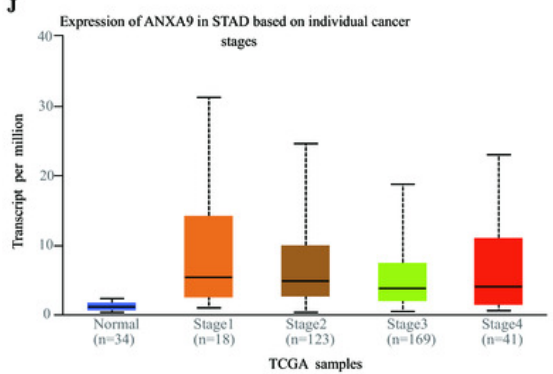


Figure 5

Figure 5

The correlation between ANXA9 expression and prognosis in GC. A-C: The K-M survival curves for OS (A), 3-year survival (B), and 5-year survival (C) of patients with GC.

A

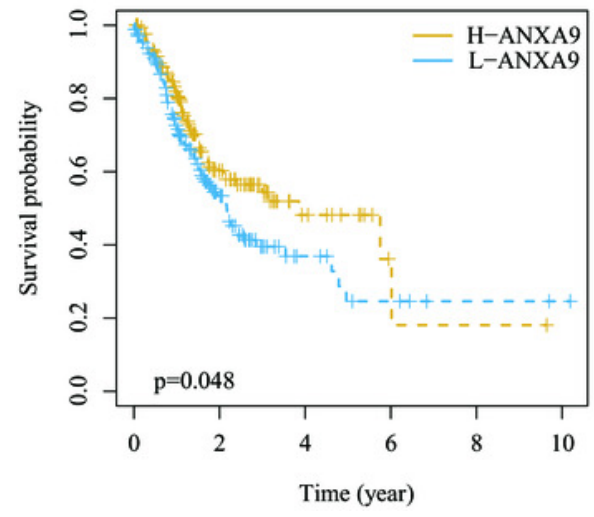

B

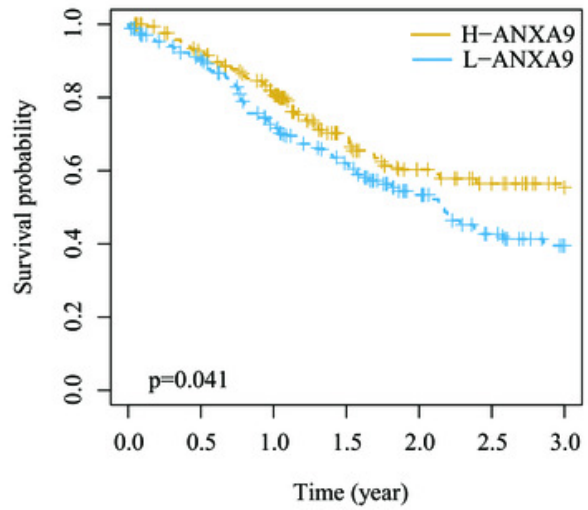

$\mathrm{C}$

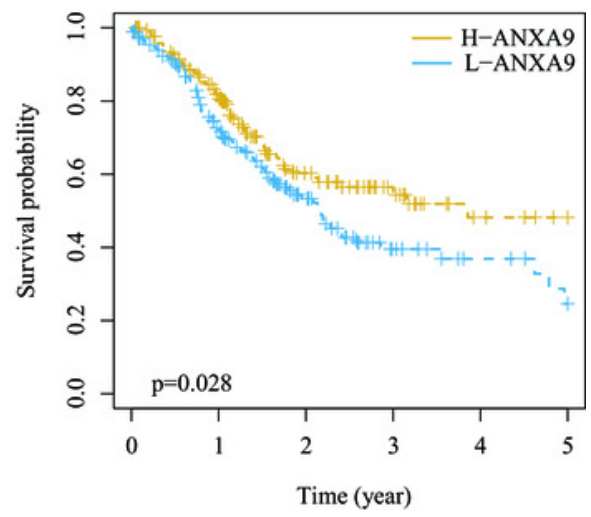


Figure 6

Figure 6

The biological functions of ANXA9 in GC. A: the GO enrichment results based on the genes in the ANXA9 high expression group. B: the GO enrichment results based on the genes in the ANXA9 low expression group.
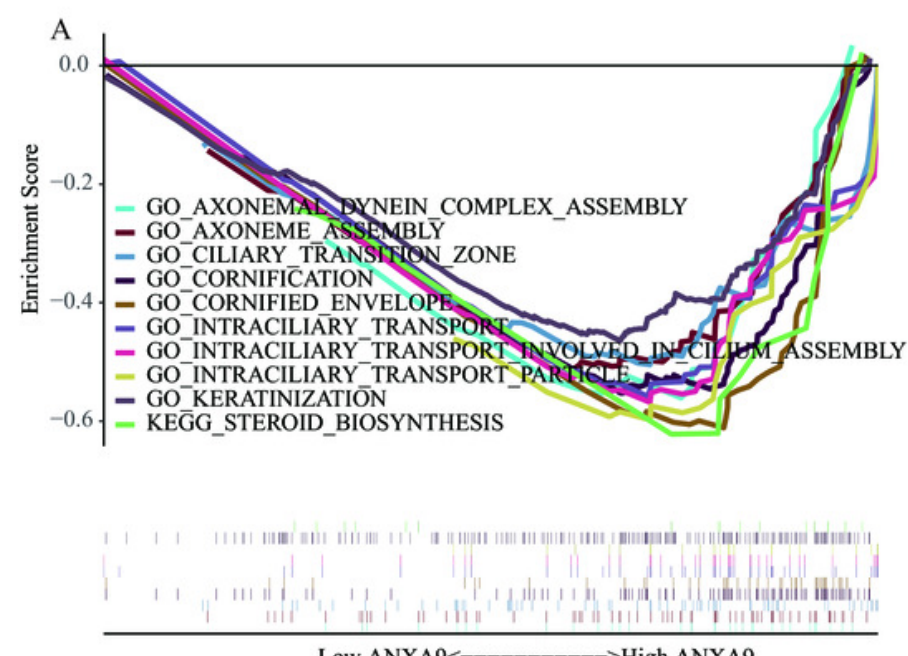
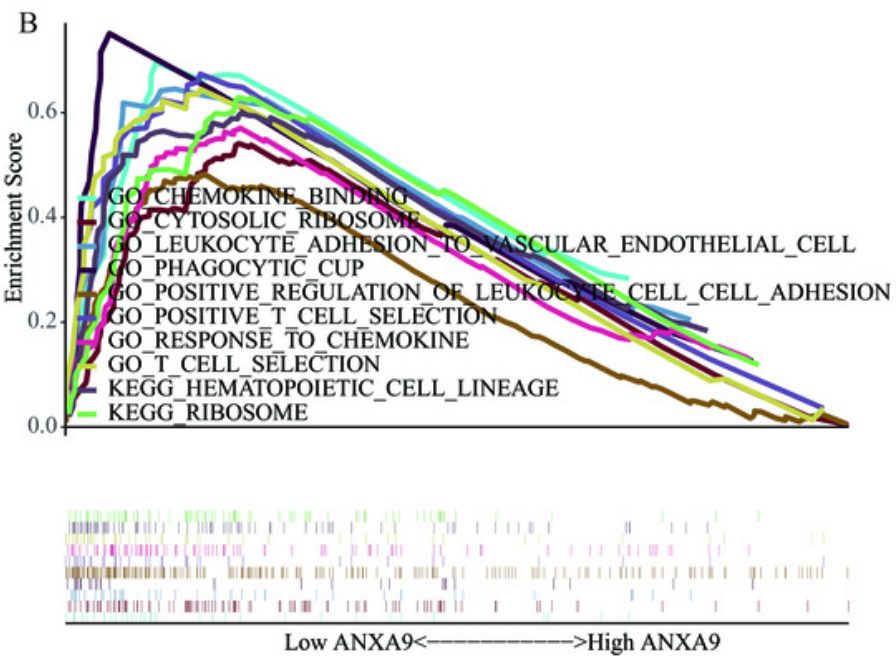
Figure 7

Figure 7

Correlation between ANXA9 expression and immune infiltration levels in GC. A: the proportion and composition of infiltrating immune cells between ANXA9 high and low expression groups based on 21 gene sets including immune cells and the activity of immunerelated pathways. B: the correlation between ANXA9 expression and immune infiltration in the TIMER database.

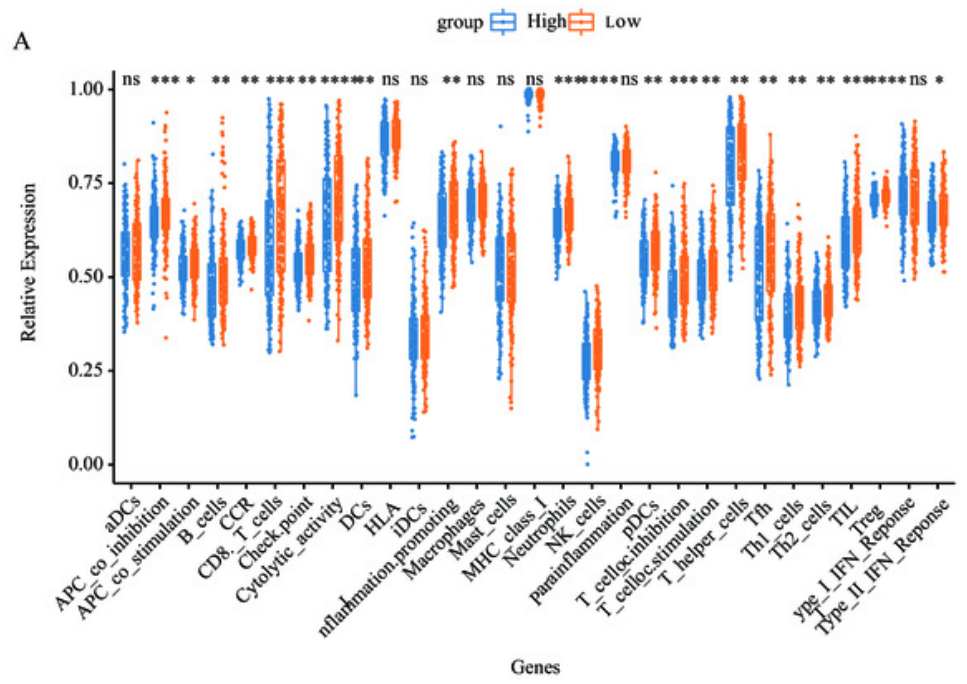

B

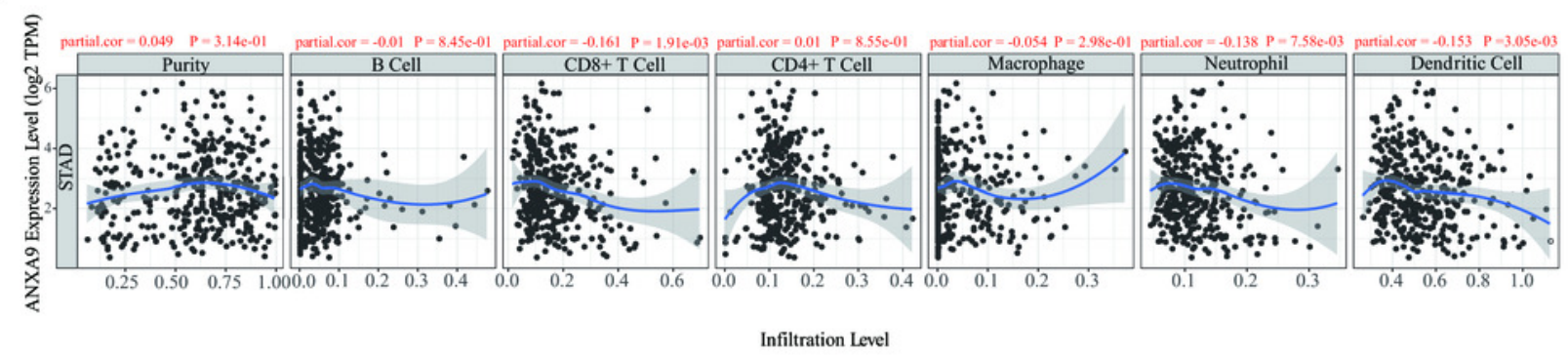


Figure 8

Figure 8

The analyses of ANXA9 DNA methylation and CNV. A-G, the correlation between DNA methylation level and expression of ANXA9 in different methylation modification sites, including cg04144222 (A), cg07337598 (B), cg07479786 (C), cg13320146 (D), cg13912599 (E), cg20437604 (F), and cg25468058 (G). H, the correlation between CNV and expression of ANXA9.
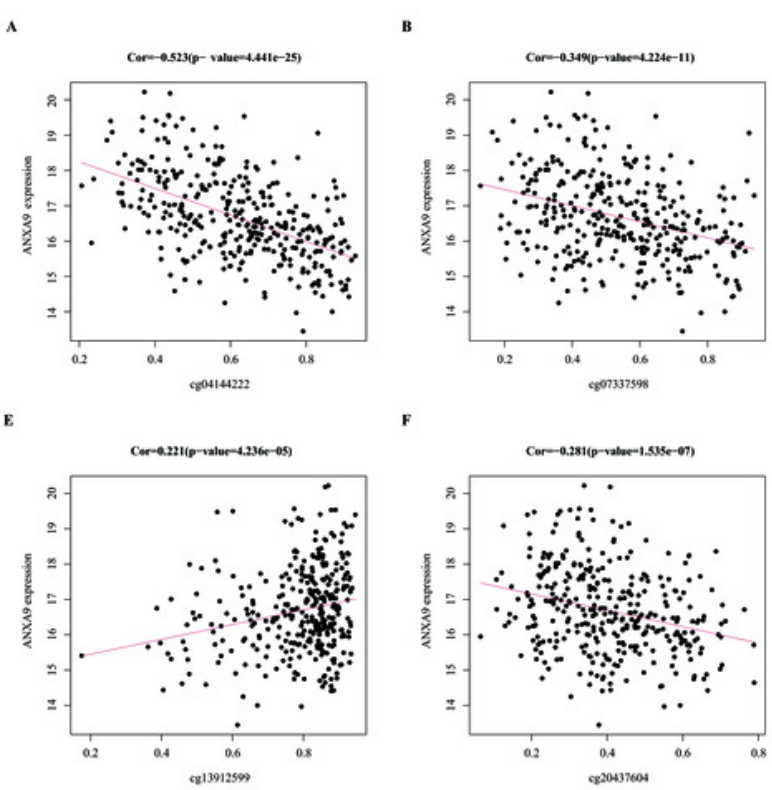

c
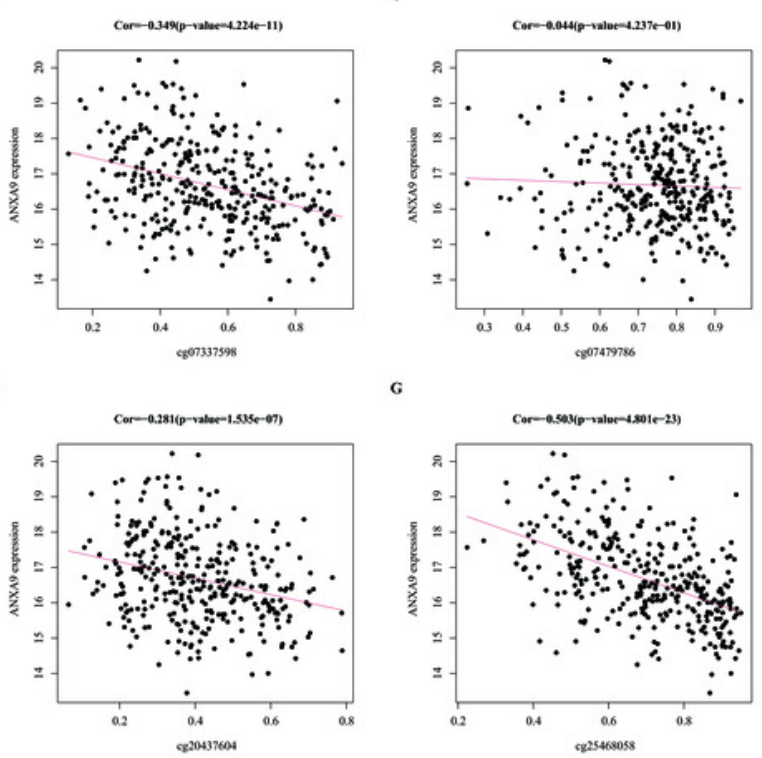

G

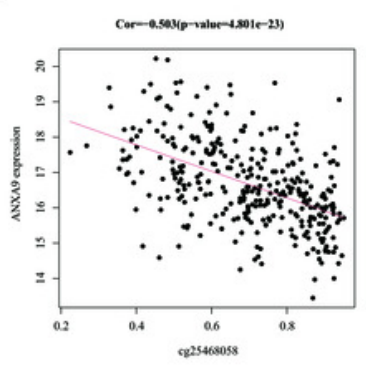

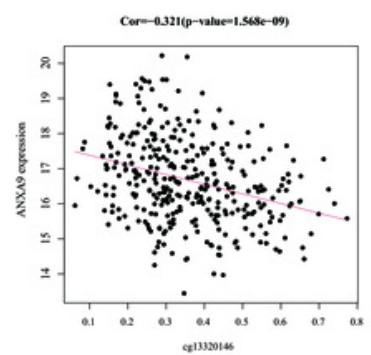

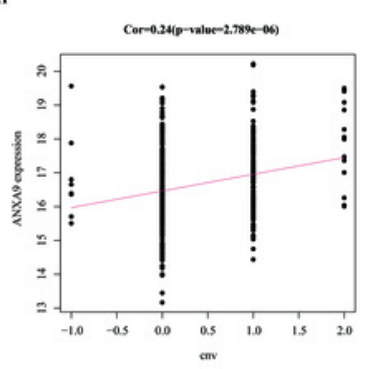


Figure 9

Figure 9

The analysis of ANXA9-related genes. A: volcano plot of the DEGs between the ANXA9 high and ANXA9 low expression groups. B: the PPI network of ANXA9 and ANXA9- related genes. C: the GO function enriched by ANXA9-related genes. 


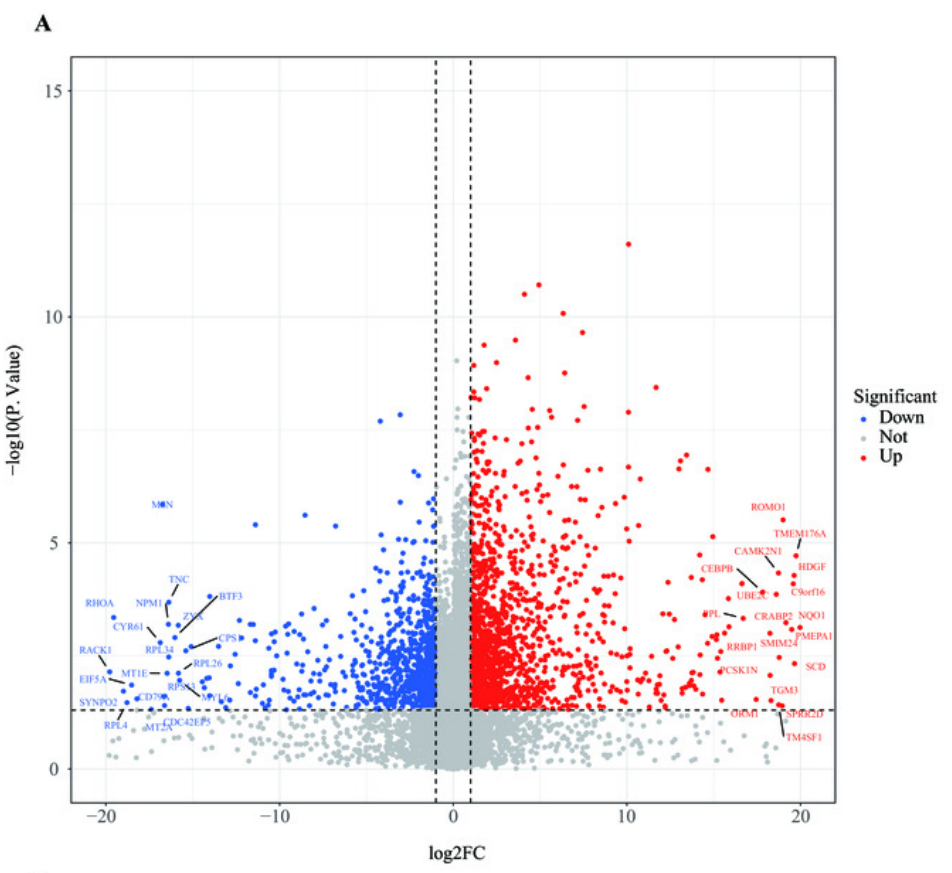

B

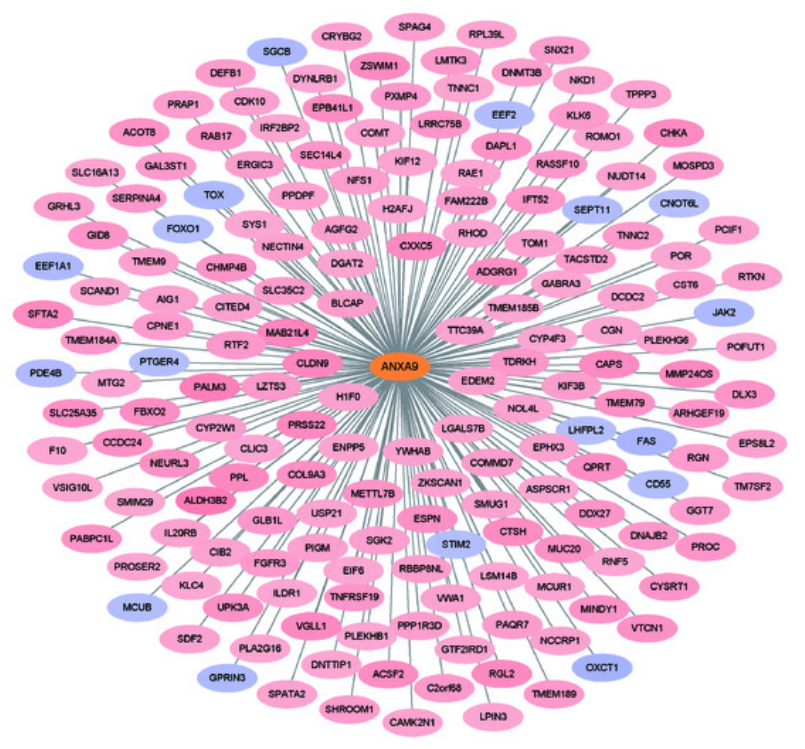

C

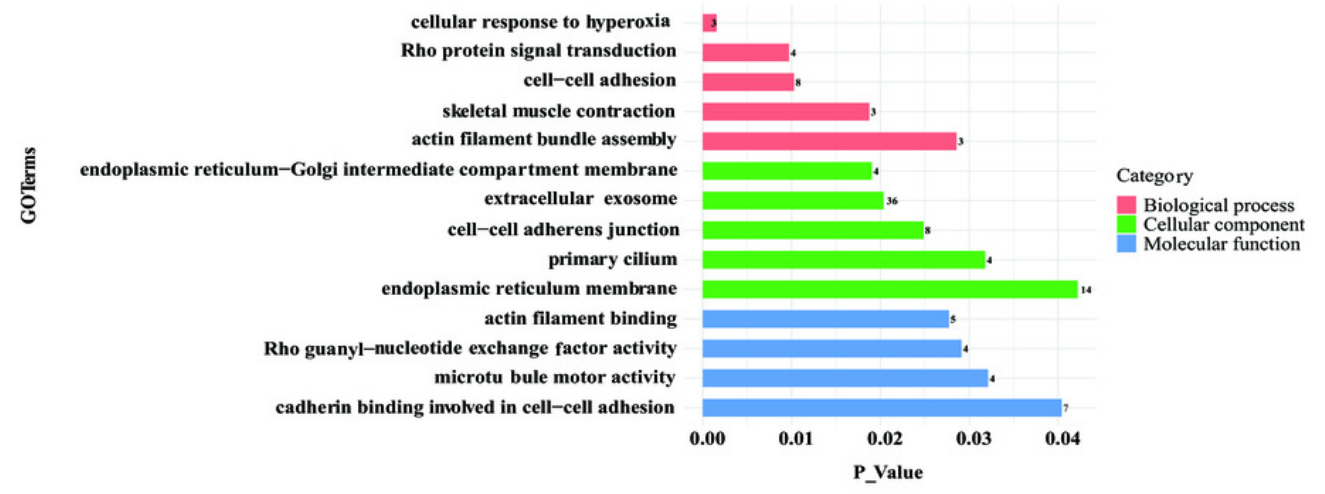

